VoL. 73 (2006) [27-36]

\title{
ON STALLINGS' UNIQUE FACTORISATION GROUPS
}

\section{Donald I. Cartwright and Bernhard Krön}

Let $\Gamma$ be a group and $\Sigma$ a symmetric generating set for $\Gamma$. In 1966, Stallings called $\Gamma$ a unique factorisation group if each group element may be written in a unique way as a product $a_{1} \ldots a_{m}$, where $a_{i} \in \Sigma$ for each $i$ and $a_{i} a_{i+1} \notin \Sigma \cup\{1\}$ for each $i<m$. In this paper we give a complete combinatorial proof of a theorem, not explicitly stated by Stallings in 1966, characterising all such pairs $(\Gamma, \Sigma)$. We also characterise the unique factorisation pairs by a certain tree-like property of their Cayley graphs.

\section{INTRODUCTION}

Let $\Gamma$ be a group and $\Sigma$ a generating set for $\Gamma$. We always assume that $\Sigma$ is symmetric $\left(\Sigma^{-1}=\dot{\Sigma}\right)$ and that $1 \notin \Sigma$. We do not assume that $\Sigma$ is finite. Let $\Sigma^{*}$ denote the set of all words over $\Sigma$. We write $\left(a_{1}, \ldots, a_{m}\right)$ for a word, and $a_{1} \ldots a_{m}$ for the group element this word represents. The empty word represents 1 . Let $L_{\Sigma}$ denote the set of the words $\left(a_{1}, \ldots, a_{m}\right) \in \Sigma^{*}$ in which $a_{i} a_{i+1} \notin \Sigma \cup\{1\}$ for all $i<m$ (together with the empty word). Given $g \in \Gamma$, any word in $\Sigma^{*}$ of minimal length representing $g$ is in $L_{\Sigma}$. Modifying [7] slightly, we call $(\Gamma, \Sigma)$ a unique factorisation pair if for each $g \in \Gamma$ there is only one word in $L_{\Sigma}$ which represents $g$. Equivalently:

DEFINITION 1: The pair $(\Gamma, \Sigma)$ is called a unique factorisation pair if the map $\left(a_{1}, \ldots, a_{m}\right) \mapsto a_{1} \ldots a_{m}$ is a bijection $L_{\Sigma} \rightarrow \Gamma$.

One goal of this paper is to describe (in Theorem 1 below), up to isomorphism, all possible groups $\Gamma$ and all possible generating sets $\Sigma$ on a given group $\Gamma$ such that $(\Gamma, \Sigma)$ is a unique factorisation pair. The relevant notion of isomorphism here is the obvious one: $\left(\Gamma_{1}, \Sigma_{1}\right)$ and $\left(\Gamma_{2}, \Sigma_{2}\right)$ are isomorphic if there is a group isomorphism $f: \Gamma_{1} \rightarrow \Gamma_{2}$ such that $f\left(\Sigma_{1}\right)=\Sigma_{2}$.

A second goal is to characterise (in Theorem 2 below) the Cayley graphs of unique factorisation pairs in terms of a "tree-like" property they have.

We start by listing some examples of unique factorisation pairs $(\Gamma, \Sigma)$. We shall check that Examples 2 and 3 are unique factorisation pairs in Lemma 2.6 below.

Received 25th July, 2005

The second author was supported by projects J2245 and P16004-N05 of the Austrian Science Fund (FWF).

Since completing this paper, we have been informed by Jean Mairesse that a revised version of [4] will contain Theorem 1, stated in the form of an algorithm, which he had independently deduced from [7].

Copyright Clearance Centre, Inc. Serial-fee code: 0004-9727/06 \$A2.00+0.00. 
ExAmple 1. Let $G$ be a group. Then $(G, G \backslash\{1\})$ is a unique factorisation pair.

EXAMPLE 2. Let $F$ be a free group, with free generators $y_{k}, k \in K$, for some set $K$. Write $y_{0}=1$. Let $\Sigma$ consist of the elements $y_{k}^{-1} y_{\ell}$, where $k, \ell \in K \cup\{0\}, k \neq \ell$. Then it is not hard to show that $(F, \Sigma)$ is a unique factorisation pair by appealing to the fact that $\left(F, \Sigma_{0}\right)$ is a unique factorisation pair, where $\Sigma_{0}=\left\{y_{k}^{ \pm 1}: k \in K\right\}$.

ExAMPLE 3. Let $G$ be a group, and let $F$ be as in Example 2. Let $\Sigma$ denote the set of elements in the free product $G * F$, other than 1 , of the form $y_{k}^{-1} g y_{\ell}$, where $g \in G$ and $k, \ell \in K \cup\{0\}$. Then $(G * F, \Sigma)$ is a unique factorisation pair (see Lemma 2.6). Examples 1 and 2 are the special cases $K^{\prime}=\emptyset$ and $G=\{1\}$, respectively, of this example.

EXAMPLE 4. Let $\left(\Gamma_{j}, \Sigma_{j}\right), j \in J$, be a family of unique factorisation pairs. Let $\Gamma$ denote the free product of the $\Gamma_{j}$ 's, and let $\Sigma$ denote the union of the sets $\Sigma_{j}$. Then $(\Gamma, \Sigma)$ is a unique factorisation pair. We shall write $(\Gamma, \Sigma)=*_{j \in J}\left(\Gamma_{j}, \Sigma_{j}\right)$.

THEOREM 1. Let $(\Gamma, \Sigma)$ be any unique factorisation pair. Then $(\Gamma, \Sigma)$ is isomorphic to a free product $*_{j \in J}\left(\Gamma_{j}, \Sigma_{j}\right)$, where each $\left(\Gamma_{j}, \Sigma_{j}\right)$ is as in Example 3.

Stallings' paper [7] is mostly concerned with "partial groups" and their "universal groups". It concludes with a terse discussion of the structure of these partial groups, with only a brief proof sketch. From this discussion, using universal groups it is not a large step to arrive at our Theorem 1, though this is not done in [7]. We feel that the result is of sufficient interest to warrant a clear statement and the complete proof we present in Section 2, particularly as there has been renewed interest in these groups amongst people studying random walks on groups; Mairesse and Mathéus $[4,5]$ were able to perform very explicit calculations concerning random walks on these groups in which the transitions are of the form $g \mapsto g a(g \in \Gamma, a \in \Sigma)$. Our proof uses several of the ideas indicated by Stallings [7], but takes a more direct combinatorial group theory approach, rather than using the methods of partial groups and their universal groups.

It follows from Theorem 1 that if $(\Gamma, \Sigma)$ is a unique factorisation pair and $\Sigma$ is finite, then $\Gamma$ is plain, that is, a free product $G_{1} * \cdots * G_{s} * F_{r}$ of finitely many finite groups $G_{1}, \ldots, G_{s}$ and a free group $F_{\mathrm{r}}$ (where $r, s \geqslant 0$ ). This much had been deduced from Stallings' results and written down explicitly by Haring-Smith [3], and used by him and others subsequently (for example, $[1,2]$ ). But Theorem 1 provides more, giving precise information about the generating set $\Sigma$. In particular, for each plain group $\Gamma$, there are, up to isomorphism, only finitely many generating sets $\Sigma$ for which $(\Gamma, \Sigma)$ is a unique factorisation pair.

EXAMPLE 5. Let $r \geqslant 1$, and let $F_{r}$ denote the free group on $r$ free generators. Given any integers $r_{1} \geqslant \cdots \geqslant r_{k} \geqslant 1$ such that $r_{1}+\cdots+r_{k}=r$, for $j=1, \ldots, k$, let $\Sigma_{\mathbf{r}_{j}}$ be the generating set on $F_{r_{j}}$ described in Example 2. Form $*_{j=1}^{k}\left(F_{r_{j}}, \Sigma_{r_{j}}\right)$, in the notation of Example 4. By Example 4, this gives a unique factorisation pair $\left(F_{r}, \Sigma\right)$. By Theorem 1, any generating set $\Sigma$ on the free group $F_{r}$ such that $\left(F_{r}, \Sigma\right)$ is a unique factorisation 
pair is of this type. Since the numbers $r_{k}$ are the sizes of the equivalence classes $[a]$ (see Lemmas 2.3 and 2.6 below), distinct partitions correspond to non-isomorphic unique factorisation pairs. So up to isomorphism, the number of distinct $\Sigma$ 's on $F_{r}$ is the number of distinct partitions of $r$. The usual generating set of $F_{r}$ (that is, a set of free generators and their inverses) corresponds to the partition $1+\cdots+1$ of $r$.

Recall that the Cayley graph $\operatorname{Cay}(\Gamma, \Sigma)$ of a group $\Gamma$ with respect to the symmetric generating set $\Sigma$, with $1 \notin \Sigma$, is the undirected graph having vertex set $V=\Gamma$, and edge set $E$ consisting of the pairs $\{g, g a\}$, where $g \in \Gamma$ and $a \in \Sigma$. If $g_{1}, g_{2} \in \Gamma$, let $d_{\Sigma}\left(g_{1}, g_{2}\right)$ denote the distance from $g_{1}$ to $g_{2}$ in $\operatorname{Cay}(\Gamma, \Sigma)$.

In Section 3, we shall characterise the Cayley graphs of unique factorisation pairs. As we shall see below, if $(\Gamma, \Sigma)$ is a unique factorisation pair, then we obtain an equivalence relation on $\Sigma$ by writing $a \sim b$ if and only if $a^{-1} b \in \Sigma \cup\{1\}$. This means that the equivalence class $[a]$ of $a$, together with 1 , spans a complete subgraph of $\operatorname{Cay}(\Gamma, \Sigma)$. Since left multiplication by group elements is a graph automorphism, the family

$$
\mathcal{C}=\{g([a] \cup\{1\}): g \in \Gamma, a \in \Sigma\}
$$

consists of non-empty subsets $C$ of $\Gamma$ with the following properties: (i) each edge $\{x, y\}$ is contained in some $C \in \mathcal{C}$, and (ii) each $C$ spans a complete subgraph.

If $X=(V, E)$ is any connected undirected graph, and if $\mathcal{C}$ is a family of non-empty subsets of $V$ with property (i), then we can form a connected graph $X_{\mathcal{C}}=\left(V_{\mathcal{C}}, E_{\mathcal{C}}\right)$ with vertex set $V \cup \mathcal{C}$ and edges $\{x, C\}$, where $C \in \mathcal{C}$ and $x \in C$. We shall show in Theorem 2 below that if $(\Gamma, \Sigma)$ is a unique factorisation pair then $\operatorname{Cay}(\Gamma, \Sigma)_{\mathcal{C}}$ is a tree, and that this property characterises unique factorisation pairs.

The Cayley graph of a unique factorisation pair $(\Gamma, \Sigma)$ has other tree-like features. As remarked in [4, Section 3.3], the removal of any vertex disconnects the graph, provided that $(\Gamma, \Sigma)$ is not as in Example 1. We shall show in Proposition 2 below that the Cayley graph $\operatorname{Cay}(\Gamma, \Sigma)$ of a unique factorisation pair $(\Gamma, \Sigma)$ is "2-bounded by a tree". Agostino [2] called a graph $X h$-bounded by a tree if it has a spanning tree $T$ such that the end points of an arbitrary edge of $X$ are at distance at most $h$ in $T$. Not every pair $(\Gamma, \Sigma)$ whose Cayley graph is 2-bounded by a tree is a unique factorisation pair. For example, $\Gamma=\mathbb{Z}, \Sigma=\{ \pm 1, \pm 2\}$.

Since any word of minimal length representing a given group element is in $L_{\Sigma}$, the evaluation map $\left(a_{1}, \ldots, a_{m}\right) \mapsto a_{1} \ldots a_{m}$ from $L_{\Sigma}$ to $\Gamma$ is surjective for any $(\Gamma, \Sigma)$. It also follows that any unique factorisation pair $(\Gamma, \Sigma)$ has the property that each $g \in \Gamma$ is represented by a unique word in $\Sigma^{*}$ of minimal length. The characterisation of the pairs $(\Gamma, \Sigma)$ with this last property remains an open problem (see [6]).

\section{Proof of Theorem 1}

Throughout this section, let $(\Gamma, \Sigma)$ be a unique factorisation pair. 
LEMмA 2.1. If $a, b, c, a b, b c \in \Sigma \cup\{1\}$ and $b \neq 1$, then $a b c \in \Sigma \cup\{1\}$.

Proof: We may assume that $a, c, a b, b c \neq 1$. If $g=a b c$ were not in $\Sigma \cup\{1\}$, then $(a b, c)$ and $(a, b c)$ would both be in $L_{\Sigma}$ and both would represent $g$.

Lemma 2.2. Let $H$ be a group, and let $f_{0}: \Sigma \cup\{1\} \rightarrow H$ be a map such that $f_{0}(1)=1$ and $f_{0}(a b)=f_{0}(a) f_{0}(b)$ whenever $a, b \in \Sigma$ and $a b \in \Sigma \cup\{1\}$. Then there is a unique group homomorphism $f: \Gamma \rightarrow H$ which extends $f_{0}$.

Proof: If $w=\left(a_{1}, \ldots, a_{m}\right) \in \Sigma^{*}$, define $f^{*}(w)=f_{0}\left(a_{1}\right) \ldots f_{0}\left(a_{m}\right)$, and define $f^{*}(\emptyset)=1$, where $\emptyset$ denotes the empty word. Now $f^{*}(w)$ depends only on the group element $w$ represents. For $w$ can be reduced to a word in $L_{\Sigma}$ representing the same element by a succession of steps $w^{\prime} \mapsto w^{\prime \prime}$ in which a subword $(a, b)$ (where $a, b \in \Sigma$ ) of $w^{\prime}$ is replaced by the word $(a b)$ of length. 1 if $a b \in \Sigma$ or by the empty word if $a b=1$. The condition of this lemma implies that $f^{*}\left(w^{\prime}\right)=f^{*}\left(w^{\prime \prime}\right)$. If $w_{1}, w_{2} \in \Sigma^{*}$ both represent $g \in \Gamma$, then the words in $L_{\Sigma}$ to which they reduce must be the same, by the unique factorisation property. So $f^{*}\left(w_{1}\right)=f^{*}\left(w_{2}\right)$. So we may define $f(g)=f^{*}(w)$ for any $w \in \Sigma^{*}$ which represents $g$. It is clear that $f$ is a group homomorphism, and is the only one which extends $f_{0}$.

Definition 2: Let $a, b \in \Sigma$. We write $a \sim b$ if $a^{-1} b \in \Sigma \cup\{1\}$.

LEMMA 2.3. The relation $\sim$ is an equivalence relation on $\Sigma$.

Proof: If $a \sim b$ and $b \sim c$, then $a^{-1} c=\left(a^{-1}\right)(b)\left(b^{-1} c\right) \in \Sigma \cup\{1\}$ by Lemma 2.1. 0

Let $X$ denote the set of distinct equivalence classes $[a]$. Write $[a] \approx[b]$ if $[a]=[b]$ or if there is a $c \in \Sigma$ such that $[a]=[c]$ and $[b]=\left[c^{-1}\right]$. Note that $[a] \approx\left[a^{-1}\right]$.

LEMMA 2.4. The relation $\approx$ is an equivalence relation on $X$.

Proof: Suppose that $[a] \approx[b]$ and $[b] \approx[c]$, with $[a] \neq[b]$ and $[b] \neq[c]$. Then there exist $u, v \in \Sigma$ such that $[a]=[u],[b]=\left[u^{-1}\right],[b]=[v]$ and $[c]=\left[v^{-1}\right]$. Then $u^{-1} \sim v$, so that $u v \in \Sigma \cup\{1\}$. If $u v=1$, then $[c]=[a]$. If $u v \neq 1$, then $u v \sim u$ and $(u v)^{-1} \sim v^{-1}$ shows that $[a]=[u v]$ and $[c]=\left[(u v)^{-1}\right]$.

LEMma 2.5. Suppose that $(\Gamma, \Sigma)$ is a unique factorisation pair. Let $\left[x_{i}\right], i \in I$, be representatives of the distinct $\approx$ classes. Let $\Sigma_{i}=\left\{b \in \Sigma:[b] \approx\left[x_{i}\right]\right\}$. Then each set $\Sigma_{i}$ is symmetric, and $\Sigma$ is the disjoint union of the sets $\Sigma_{i}$. Let $\Gamma_{i}$ denote the subgroup of $\Gamma$ generated by $\Sigma_{i}$. Then $\left(\Gamma_{i}, \Sigma_{i}\right)$ is a unique factorisation pair, and $(\Gamma, \Sigma)$ is isomorphic to the free product $*_{i \in I}\left(\Gamma_{i}, \Sigma_{i}\right)$.

Proof: If $b \in \Sigma_{i}$, then $\left[b^{-1}\right] \approx[b] \approx\left[x_{i}\right]$, and so $b^{-1} \in \Sigma_{i}$, and $\Sigma_{i}$ is symmetric. It is clear that the sets $\Sigma_{i}$ are pairwise disjoint and have union $\Sigma$.

Let $w=\left(a_{1}, \ldots, a_{m}\right) \in L_{\Sigma_{i}}$. If $j<m$, then $a_{j} a_{j+1} \neq 1$. If $a_{j} a_{j+1} \in \Sigma$, then $a_{j} a_{j+1} \sim a_{j}$, so that $\left[a_{j} a_{j+1}\right]=\left[a_{j}\right] \approx\left[x_{i}\right]$. Hence $a_{j} a_{j+1} \in \Sigma_{i}$, contradicting $w \in L_{\Sigma_{i}}$. So $L_{\Sigma_{i}} \subset L_{\Sigma}$, and from this it is clear that $\left(\Gamma_{i}, \Sigma_{i}\right)$ is a unique factorisation pair. 
To see that $(\Gamma, \Sigma)$ is isomorphic to the free product $*_{i \in I}\left(\Gamma_{i}, \Sigma_{i}\right)$, regard $\Sigma \cup\{1\}$ $=\{1\} \cup \bigcup_{i \in I} \Sigma_{i}$ as a subset of $*_{i \in I} \Gamma_{i}$ in the usual way. Let $f_{0}: \Sigma \cup\{1\} \rightarrow *_{i \in I} \Gamma_{i}$ denote the inclusion map. Let us check the condition of Lemma 2.2. Suppose that $a, b \in \Sigma$ and that $a b \in \Sigma \cup\{1\}$. If $a b=1$ and $a \in \Sigma_{i}$, then $b=a^{-1} \in \Sigma_{i}$ too, and $f_{0}(a) f_{0}(b)=a b$ $=1=f_{0}(a b)$. If $a b \in \Sigma$ and $a \in \Sigma_{i}$, then $a b \sim a$ shows that $[a b]=[a] \approx\left[x_{i}\right]$ and $a b \in \Sigma_{i}$. So $(a b)^{-1} \in \Sigma_{i}$, and $b^{-1} \sim b^{-1} a^{-1}$ shows that $\left[b^{-1}\right]=\left[(a b)^{-1}\right] \approx\left[x_{i}\right]$. Thus $b^{-1} \in \dot{\Sigma}_{i}$, and so $b \in \Sigma_{i}$ too. So $a, b, a b \in \Sigma_{i}$, and $f_{0}(a) f_{0}(b)=a b=f_{0}(a b)$. So by Lemma 2.2, there is a unique homomorphism $f: \Gamma \rightarrow *_{i \in I} \Gamma_{i}$ extending $f_{0}$.

Let $\Sigma^{\prime}=\bigcup_{i \in I} \Sigma_{i}$, regarded as a subset of $*_{i \in I} \Gamma_{i}$. It is evident (Example 4) that $\left(*_{i \in I} \Gamma_{i}, \Sigma^{\prime}\right)$ is a unique factorisation pair. Let $h_{0}: \Sigma^{\prime} \cup\{1\} \rightarrow \Gamma$ map each $a \in \Sigma_{i}$, regarded as an element of the free product, to $a$, regarded as an element of $\Gamma$. Clearly $h_{0}$ satisfies the condition of Lemma 2.2. The unique extension $h$ of $h_{0}$ to $*_{i \in I} \Gamma_{i}$ is the inverse of $f$, because of the uniqueness in Lemma 2.2, since $h_{0} \circ f_{0}$ is the identity on $\Sigma$ and $f_{0} \circ h_{0}$ is the identity on $\Sigma^{\prime}$. Notice that $f(\Sigma)=\Sigma^{\prime}$, and so $f$ is an isomorphism of unique factorisation pairs.

LEMma 2.6. The pair $(G * F, \Sigma)$ of Example 3 is a unique factorisation pair, and for any $a, b \in \Sigma$ we have $[a] \approx[b]$. If $G=\{1\}$ and $|K|=r<\infty$, then each $[a]$ has $r$ elements.

Proof: A proof that $(G * F, \Sigma)$ is a unique factorisation pair can be given by showing that $G * F$ is a universal group of the partial group $\Sigma$, and appealing to [7, Proposition 2.2]. We shall instead give a more direct proof.

Let $\Sigma_{0}=(G \backslash\{1\}) \cup\left\{y_{k}^{ \pm 1}: k \in K\right\}$. By the uniqueness of normal forms in a free product and by the evident fact that $\left(F,\left\{y_{k}^{ \pm 1}: k \in K\right\}\right)$ is a unique factorisation pair, it is clear that $\left(G * F, \Sigma_{0}\right)$ is a unique factorisation pair.

Let $w=\left(a_{1}, \ldots, a_{m}\right) \in L_{\Sigma}$ represent $g \in G * F$. For each $i$, write $a_{i}=u_{i}^{-1} g_{i} v_{i}$, where $g_{i} \in G$ and $u_{i}, v_{i} \in\left\{y_{k}: k \in K \cup\{0\}\right\}$ for each $i$. If $i<m$ then $v_{i} u_{i+1}^{-1} \neq 1$, since otherwise $a_{i} a_{i+1}=u_{i}^{-1} g_{i} g_{i+1} v_{i+1}$ is in $\Sigma \cup\{1\}$. From $w$, form the word $\widetilde{w}$ $=\left(u_{1}^{-1}, g_{1}, v_{1}, u_{2}^{-1}, g_{2}, \ldots, g_{m}, v_{m}\right)$, which has letters in $\Sigma_{0} \cup\{1\}$. Let $w_{0}=\left(x_{1}, \ldots, x_{\ell}\right)$ be the word obtained from $\widetilde{w}$ by discarding all 1's. We claim that $w_{0} \in L_{\Sigma_{0}}$. Notice that if $a, b \in \Sigma_{0}$ and also $a b \in \Sigma_{0}$, then $a, b$ must both be in $G \backslash\{1\}$. So if $x_{i} x_{i+1} \in \Sigma_{0}$ for some $i<\ell$, then $x_{i}=g_{j}, v_{j}=1, u_{j+1}^{-1}=1$ and $x_{i+1}=g_{j+1}$ for some $j<m$. But this is impossible because $v_{j} u_{j+1}^{-1} \neq 1$. If instead $x_{i} x_{i+1}=1$, then the pair $\left(x_{i}, x_{i+1}\right)$ is either $\left(y_{k}, y_{k}^{-1}\right)$ or $\left(y_{k}^{-1}, y_{k}\right)$ for some $k$, or $\left(g, g^{-1}\right)$ for some $g \in G$, and these possibilities are all excluded because $a_{j} \neq 1$ and $a_{j} a_{j+1} \notin \Sigma \cup\{1\}$ for each $j$.

Notice that the first letter $x_{1}$ of $w_{0}$ is either $u_{1}^{-1}$ (if $u_{1} \neq 1$ ), $g_{1}$ (if $u_{1}=1$ and $g_{1} \neq 1$ ) or $v_{1}$ (if $u_{1}=1$ and $g_{1}=1$ ).

Suppose that there is an element $g \in G * F$ which is represented by two distinct words, $w=\left(a_{1}, \ldots, a_{m}\right)$ and $w^{\prime}=\left(a_{1}^{\prime}, \ldots, a_{m^{\prime}}^{\prime}\right)$ in $L_{\Sigma}$. Write $a_{i}=u_{i}^{-1} g_{i} v_{i}$ and $a_{j}^{\prime}=u_{j}^{\prime-1} g_{j}^{\prime} v_{j}^{\prime}$ as 
above. Starting from $w$ and $w^{\prime}$, we get words $w_{0}=\left(x_{1} \ldots, x_{\ell}\right)$ and $w_{0}^{\prime}=\left(w_{1}, \ldots, w_{\ell^{\prime}}^{\prime}\right)$ in $L_{\Sigma_{0}}$ as above. Choose such a $g$ so that the number $\ell+\ell^{\prime}$ of $u_{i}$ 's, $g_{i}$ 's, et cetera, which are not 1 is minimal. By the unique factorisation property of $\left(G * F, \Sigma_{0}\right)$, we have $w_{0}=w_{0}^{\prime}$. By considering the first letter $x_{1}$ of $w_{0}$, we see that $u_{1} \neq 1$ if and only if $u_{1}^{\prime} \neq 1$, in which case $u_{1}=u_{1}^{\prime}$. Cancelling $u_{1}^{-1}$ from both $a_{1}$ and $a_{1}^{\prime}$, we find that $u_{1} g$ is represented by two distinct words in $L_{\Sigma}$ in which the total number of $u_{i}$ 's, et cetera, is smaller than in $w$ and $w^{\prime}$, contradicting the minimality in our choice of $g$. The same contradiction is reached in the case $u_{1}=u_{1}^{\prime}=1$ and $g_{1}, g_{1}^{\prime} \neq 1$ and in the case $u_{1}=u_{1}^{\prime}=1, g_{1}=g_{1}^{\prime}=1$ and $v_{1}, v_{1}^{\prime} \neq 1$. So $(G * F, \Sigma)$ is a unique factorisation pair.

If $G \neq\{1\}$, fix $g_{0} \in G \backslash\{1\}$. If $a \in \Sigma$ has the form $g y_{\ell}$, then $[a]=[g]=\left[g_{0}\right]$. If instead $a=y_{k}^{-1} g y_{\ell}$ where $k \in K$, then $\left[g_{0}\right]=\left[y_{k}\right]$ and $[a]=\left[y_{k}^{-1}\right]$, so that again $[a] \approx\left[g_{0}\right]$. If $G=\{1\}$ but $K \neq \emptyset$, fix $k_{1} \in K$. If $a=y_{\ell}$ for some $\ell \in K$, then $[a]=\left[y_{k_{1}}\right]$. If $a=y_{k}^{-1} y_{\ell}$ with $k \neq 0$, then $[a]=\left[y_{k}^{-1}\right]$ and $\left[y_{k_{1}}\right]=\left[y_{k}\right]$, so that again $[a] \approx\left[y_{k_{1}}\right]$. Since $y_{\ell}^{-1} y_{k} y_{k_{1}} \notin \Sigma \cup\{1\}, a \notin\left[y_{k_{1}}\right]$ in the second case. Hence $\left[y_{k_{1}}\right]$ has exactly $r$ elements if $|K|=r<\infty$. Similarly, each $\left[y_{k}^{-1}\right]$ has $r$ elements.

By Lemma 2.5 , to prove Theorem 1 we may suppose that $[a] \approx[b]$ for all $a, b \in \Sigma$. Fix $a_{0} \in \Sigma$. We can choose representatives $x_{k}^{-1}(k \in K$, say) of the distinct classes $[a]$ other than $\left[a_{0}\right]$ such that $\left[a_{0}\right]=\left[x_{k}\right]$ for each $k$. We also write $x_{0}=1$.

LEMMA 2.7. The set $\Pi=\{1\} \cup\left\{a \in \Sigma: a \sim a_{0}\right.$ and $\left.a^{-1} \sim a_{0}\right\}$ is a subgroup of $\Gamma$.

Proof: Suppose that $a, b \in \Pi \backslash\{1\}$. Then $a^{-1} \sim a_{0} \sim b$ and so $a b \in \Sigma \cup\{1\}$. If $a b \neq 1$, then $a b \in \Sigma$ and $a b \sim a \sim a_{0}$ and $(a b)^{-1} \sim b^{-1} \sim a_{0}$. So $a b \in \Pi$.

LEMMA 2.8. Each $a \in \Sigma$ can be written in a unique way as a product $x_{k}^{-1} g x_{\ell}$, where $k, \ell \in K \cup\{0\}$ and $g \in \Pi$. Moreover, $[a]=\left[x_{k}^{-1}\right]$ if $a \not a_{0}$ and $\left[a^{-1}\right]=\left[x_{\ell}^{-1}\right]$ if $a^{-1} \not a_{0}$.

Proof: If $a \in \Pi$, take $k=\ell=0$ and $g=a$. If $a \in \Sigma$ satisfies $a \sim a_{0}$ but $a^{-1} \nsim a_{0}$, then there is an $\ell \in K$ such that $\left[a^{-1}\right]=\left[x_{\ell}^{-1}\right]$. Write $g=a x_{\ell}^{-1}$. Then $a=g x_{\ell}=x_{0}^{-1} g x_{\ell}$. Also, $g \sim a \sim a_{0}$ and $g^{-1} \sim x_{\ell} \sim a_{0}$ shows that $g \in \Pi$. Similarly, if $a \not a_{0}$ and $a^{-1} \sim a_{0}$, then writing $[a]=\left[x_{k}^{-1}\right]$, we have $a=x_{k}^{-1} g x_{0}$ for some $g \in \Pi$.

If $a \in \Sigma$ and $a, a^{-1} \not a_{0}$, then $[a]=\left[x_{k}^{-1}\right]$ and $\left[a^{-1}\right]=\left[x_{\ell}^{-1}\right]$ for some $k, \ell \in K$. Then $a, x_{k}, x_{\ell}, x_{k} a$ and $a x_{\ell}^{-1}$ are in $\Sigma \cup\{1\}$ and $a \neq 1$. Hence $x_{k} a x_{\ell}^{-1}=\left(x_{k} a\right)\left(a^{-1}\right)\left(a x_{\ell}^{-1}\right)$ $\in \Sigma \cup\{1\}$ by Lemma 2.1. Write $x_{k} a x_{\ell}^{-1}=g$. Then $a=x_{k}^{-1} g x_{\ell}$. Also, $g \in \Pi$. For $x_{k}^{-1} g=a x_{\ell}^{-1} \in \Sigma \cup\{1\}$ shows that $g \sim x_{k} \sim a_{0}$, and $g x_{\ell}=x_{k} a \in \Sigma \cup\{1\}$ shows that $g^{-1} \sim x_{\ell} \sim a_{0}$. If also $a=x_{k^{\prime}}^{-1} g^{\prime} x_{\ell^{\prime}}$, and $k^{\prime} \neq 0$, then $x_{k^{\prime}} a=g^{\prime} x_{\ell^{\prime}} \in \Sigma \cup\{1\}$ because $g^{\prime} \sim a_{0} \sim x_{\ell^{\prime}}$ if $g^{\prime} \neq 1$ and $\ell^{\prime} \neq 0$, while $x_{k^{\prime}} a=g^{\prime} x_{\ell^{\prime}} \in \Sigma \cup\{1\}$ is clear if $g^{\prime}=1$ or $\ell^{\prime}=0$. Thus $\left[x_{k}^{-1}\right]=[a]=\left[x_{k^{\prime}}^{-1}\right]$ and $k^{\prime}=k$. If $k^{\prime}=0$, then $a=g^{\prime} x_{\ell^{\prime}} \sim g^{\prime} \sim a_{0}$, contrary to hypothesis. Similarly $\ell^{\prime}=\ell$, and therefore $g^{\prime}=g$. The uniqueness when $a \sim a_{0}$ or $a^{-1} \sim a_{0}$ is shown in a similar way. 
Proposition 1. Suppose that $(\Gamma, \Sigma)$ is a unique factorisation pair and that $a_{0} \in \Sigma$, with $[a] \approx\left[a_{0}\right]$ for all $a \in \Sigma$. Let $\Pi$ and $\left\{x_{k}: k \in K\right\}$ be as above. Let $F$ denote the subgroup of $\Gamma$ generated by the $x_{k}$ 's. Then $F$ is a free group, with free generators $x_{k}$, $k \in K$, and the pair $(\Gamma, \Sigma)$ is isomorphic to the pair of Example 3, where $G=\Pi$ and the $y_{k}$ 's there are the $x_{k}$ 's.

Proof: Let $F^{\prime}$ be a free group on a set $\left\{y_{k}: k \in K\right\}$ of free generators in one to one correspondence with $\left\{x_{k}: k \in K\right\}$. Let $y_{0}=1$. Let $\Sigma^{\prime}$ denote the generating set of Example 3, where $G$ and $F$ there are $\Pi$ and $F^{\prime}$. Define a function $f_{0}: \Sigma \rightarrow \Pi * F^{\prime}$ by writing each $a \in \Sigma$ in its unique form $x_{k}^{-1} g x_{\ell}(k, \ell \in K \cup\{0\}, g \in \Pi)$, and defining $f_{0}(a)=y_{k}^{-1} g y_{\ell}$, regarded as an element of the free product. We now check the condition of Lemma 2.2 .

Suppose that $a, b \in \Sigma$ and that $a b \in \Sigma \cup\{1\}$. Write $a=x_{k}^{-1} g x_{\ell}$ and $b=x_{m}^{-1} g^{\prime} x_{n}$ as in Lemma 2.8. Assume first that $\ell, m \neq 0$. Then $x_{\ell}^{-1} \sim a^{-1} \sim b \sim x_{m}^{-1}$, and so $\ell=m$ and $a b=x_{k}^{-1} g g^{\prime} x_{n}$. If $\ell \neq 0$ and $m=0$, then $x_{\ell}^{-1} \sim a^{-1} \sim b=g^{\prime} x_{n} \sim a_{0}$, which is impossible. Similarly, if $\ell=0$ and $m \neq 0$, then $a_{0} \sim g^{-1} x_{k}=a^{-1} \sim b \sim x_{m}^{-1}$ is impossible. Finally, if $\ell=0=m$, then $a b=x_{k}^{-1} g g^{\prime} x_{n}$. Thus in all cases, $\ell=m$ and $a b=x_{k}^{-1} g g^{\prime} x_{n}$. So $f_{0}(a) f_{0}(b)=\left(y_{k}^{-1} g y_{\ell}\right)\left(y_{m}^{-1} g^{\prime} y_{n}\right)=y_{k}^{-1} g g^{\prime} y_{n}=f_{0}(a b)$. By Lemma $2.2, f_{0}$ lifts uniquely to a group homomorphism $f: \Gamma \rightarrow \Pi * F^{\prime}$. Notice that $f(\Sigma)=\Sigma^{\prime}$.

Let $h_{0}: \Sigma^{\prime} \rightarrow \Gamma$ denote the map which sends each $y_{k}^{-1} g y_{\ell}$ to $x_{k}^{-1} g x_{\ell} \in \Gamma$. As before, one checks that it satisfies the conditions of Lemma 2.2. Since $\left(\Pi * F^{\prime}, \Sigma^{\prime}\right)$ is a unique factorisation pair by Lemma 2.6, $h_{0}$ extends to a group homomorphism $h: \Pi * F^{\prime} \rightarrow \Gamma$. Since $f_{0}$ and $h_{0}$ are mutually inverse bijections between $\Sigma$ and $\Sigma^{\prime}$, their extensions are mutually inverse group isomorphisms, by the uniqueness part of Lemma 2.2 .

\section{CAYLEY GRAPHS OF UNIQUE FACTORISATION PAIRS}

Throughout this section, let $(\Gamma, \Sigma)$ denote a unique factorisation pair, and let $X=\operatorname{Cay}(\Gamma, \Sigma)$ be its Cayley graph.

LEMma 3.1. If $a \in \Sigma$, then $a\left(\left[a^{-1}\right] \cup\{1\}\right)=[a] \cup\{1\}$.

Proof: Let $b \in\left[a^{-1}\right] \cup\{1\}$. We claim that $a b \in[a] \cup\{1\}$. This is clear if $b=1$ or $b=a^{-1}$, so assume otherwise. Then $b \sim a^{-1}$ and so $a b \in \Sigma$. Also, $a^{-1}(a b)=b \in \Sigma$, and so $a b \in[a]$. Hence $a\left(\left[a^{-1}\right] \cup\{1\}\right) \subset[a] \cup\{1\}$. Replacing $a$ by $a^{-1}$, we get the reverse inclusion.

The fact that $\sim$ is an equivalence relation implies that each set in the family (1.1) spans a complete subgraph of $X$. The vertices $g, g a$ of any edge in $X$ are both contained in $g([a] \cup\{1\}) \in \mathcal{C}$. The next lemma shows that this is the only $C \in \mathcal{C}$ containing them both.

LeMma 3.2. If $C, D \in \mathcal{C}$ and $|C \cap D| \geqslant 2$, then $C=D$. 
Proof: If $g \in \Gamma \backslash\{1\}, a \in \Sigma$ and $1 \in g([a] \cup\{1\})$, then $g^{-1} \in[a]$. So by Lemma 3.1, $g([a] \cup\{1\})=g\left(\left[g^{-1}\right] \cup\{1\}\right)=[g] \cup\{1\}$. Thus the only sets in $\mathcal{C}$ containing 1 are the sets $[a] \cup\{1\}, a \in \Sigma$. Now let $C, D \in \mathcal{C}$ with $|C \cap D| \geqslant 2$. Picking any $g \in C \cap D$, let $C^{\prime}=g^{-1} C$ and $D^{\prime}=g^{-1} D$. Then $1 \in C^{\prime}, D^{\prime} \in \mathcal{C}$, and so $C^{\prime}=[a] \cup\{1\}$ and $D^{\prime}=[b] \cup\{1\}$ for some $a, b \in \Sigma$. Since $\left|C^{\prime} \cap D^{\prime}\right| \geqslant 2$, there is a $c \neq 1$ in $C^{\prime} \cap D^{\prime}$. Hence $a \sim c \sim b$, so that $[a]=[b], C^{\prime}=D^{\prime}$ and $C=D$.

Recall that a circuit in a graph is a path $\left(x_{0}, x_{1}, \ldots, x_{n}\right)$ whose vertices are distinct except that $x_{n}=x_{0}$. We shall always assume that $n \geqslant 3$.

Lemma 3.3. Let $\left(g_{0}, g_{1}, \ldots, g_{n}=g_{0}\right)$ be a circuit in $X$. Then there is a $C \in \mathcal{C}$ which contains each $g_{i}$. If $S \subset \Gamma$ spans a complete subgraph of $X$, then $S \subset C$ for some $C \in \mathcal{C}$.

Proof: Let $\pi=\left(g_{0}, g_{1}, \ldots, g_{n}=g_{0}\right)$ be a shortest circuit which is not contained in any $C \in \mathcal{C}$. If $n=3$, then $g_{0}^{-1} g_{1}, g_{1}^{-1} g_{2}, g_{2}^{-1} g_{0} \in \Sigma$, and so $g_{0}^{-1} g_{2}$ and $g_{0}^{-1} g_{1}$ are $\sim$-equivalent. Hence $g_{0}, g_{1}, g_{2} \in g_{0}\left(\left[g_{0}^{-1} g_{1}\right] \cup\{1\}\right)$. So $n \geqslant 4$ must hold.

If $g_{i}$ and $g_{i+2}$ are adjacent in $X$ for some $i \leqslant n-2$, then $\left(g_{0}, \ldots, g_{i}, g_{i+2}, \ldots, g_{n}=g_{0}\right)$ and $\left(g_{i}, g_{i+1}, g_{i+2}, g_{i}\right)$ are circuits in $X$ which are shorter than $\pi$, and so must be contained in distinct $C, D \in \mathcal{C}$. But $g_{i}, g_{i+2} \in C \cap D$, and so Lemma 3.2 shows that $C=D$, a contradiction.

So $g_{i}$ and $g_{i+2}$ are not adjacent in $X$ for any $i \leqslant n-2$. So $\left(g_{i}^{-1} g_{i+1}\right)\left(g_{i+1}^{-1} g_{i+2}\right) \notin \Sigma \cup\{1\}$ for each $i \leqslant n-2$. This shows that the word $\left(g_{0}^{-1} g_{1}, \ldots, g_{n-1}^{-1} g_{n}\right)$ is in $L_{\Sigma}$, even though it represents $g_{0}^{-1} g_{n}=1$. This contradicts the unique factorisation property, and so any circuit in $X$ is contained in some $C \in \mathcal{C}$.

Now let $S \subset \Gamma$ span a complete subgraph of $X$. We may assume that $|S| \geqslant 3$. Pick any $s_{0}, s_{1} \in S$ and $C \in \mathcal{C}$ containing $s_{0}, s_{1}$. If $S \not \subset C$, pick $s_{2} \in S \backslash C$. As $S$ spans a complete subgraph, $s_{0}, s_{1}, s_{2}$ are the vertices of a circuit, and so there is a $D \in \mathcal{C}$ containing them all. Then $s_{0}, s_{1} \in C \cap D$, and so $D=C$ by Lemma 3.2. This shows that $s_{2} \in C$, a contradiction. So $S$ must be contained in $C$.

Definition 3: Let $X=(V, E)$ be any undirected connected graph and let $\mathcal{C}$ be a family of non-empty sets of vertices such that (i) for each edge $\{x, y\}$, there is a $C \in \mathcal{C}$ such that $x, y \in C$, and (ii) each $C \in \mathcal{C}$ spans a complete subgraph of $X$. We define a graph $X_{\mathcal{C}}$ by setting $V_{\mathcal{C}}=\mathcal{C} \cup V$ and $E_{\mathcal{C}}=\{\{x, C\}: C \in \mathcal{C}, x \in C\}$.

It is easy to see that $X_{\mathcal{C}}$ is connected (hypothesis (ii) is not needed for this).

THEOREM 2. If $(\Gamma, \Sigma)$ is a unique factorisation pair, and if

$$
\mathcal{C}=\{g([a] \cup\{1\}): g \in \Gamma, a \in \Sigma\}
$$

is the family defined above, then $\operatorname{Cay}(\Gamma, \Sigma)_{\mathcal{c}}$ is a tree. Conversely, if $\Gamma$ is a group generated by a finite symmetric subset $\Sigma$, with $1 \notin \Sigma$, and if Cay $(\Gamma, \Sigma)_{\mathcal{c}}$ is a tree for some family $\mathcal{C}$, then $(\Gamma, \Sigma)$ is a unique factorisation pair. 
Proof: Suppose there is a circuit in $\operatorname{Cay}(\Gamma, \Sigma)_{\mathcal{c}}$. Then there is also a circuit of the form

$$
\pi=\left(g_{0}, C_{1}, g_{1}, C_{2}, \ldots, C_{n}, g_{n}=g_{0}\right) \text {, }
$$

where $n \geqslant 2$ and $g_{i} \in \Gamma$ and $C_{i} \in \mathcal{C}$ for each $i$. The elements $g_{i}^{-1} g_{i+2}, 0 \leqslant i \leqslant n-2$, cannot be in $\Sigma$. For otherwise $g_{i}, g_{i+1}$ and $g_{i+2}$ would be pairwise adjacent, and so in $C$ for some $C \in \mathcal{C}$, by Lemma 3.3. But $\left|C_{i+1} \cap C\right|,\left|C_{i+2} \cap C\right| \geqslant 2$, so that $C_{i+1}=C=C_{i+2}$ by Lemma 3.2. This contradicts $\pi$ being a circuit. Thus the word $\left(g_{0}^{-1} g_{1}, g_{1}^{-1} g_{2}, \ldots, g_{n-1}^{-1} g_{n}\right)$ is in $L_{\Sigma}$, is nonempty, and represents 1 , contradicting the unique factorisation property.

Conversely, suppose that $X=\operatorname{Cay}(\Gamma, \Sigma)$ is a Cayley graph and that $X_{\mathcal{C}}$ is a tree. Let $g \in \Gamma$ and let $\left(a_{1}, a_{2}, \ldots, a_{m}\right) \in L_{\Sigma}$ represent $g$. For $i=1, \ldots, m$, the vertices $a_{1} \ldots a_{i-1}$ and $a_{1} \ldots a_{i}$ are adjacent in $X$ and so are contained in some $A_{i} \in \mathcal{C}$. Then

$$
\pi=\left(a_{0}=1, A_{1}, a_{1}, A_{2}, a_{1} a_{2}, \ldots, A_{m}, a_{1} a_{2} \ldots a_{m}\right)
$$

is a path $\left(u_{0}, u_{1}, \ldots, u_{2 m}\right)$ in $X_{\mathcal{C}}$. We claim that $\pi$ is a geodesic. Since $X_{\mathcal{C}}$ is a tree, it is enough to show that $u_{k} \neq u_{k+2}$ for each $k \leqslant 2 m-2$. So suppose that $u_{k}=u_{k+2}$. If $k=2 i$, then $a_{1} \ldots a_{i-1}=a_{1} \ldots a_{i}$, which is impossible, as $a_{i} \neq 1$. If $k=2 i+1$, then $A_{i+1}=A_{i+2}=C$, say, and so $a_{1} \ldots a_{i}, a_{1} \ldots a_{i+1}, a_{1} \ldots a_{i+2}$ are all in $C$. Now $C$ spans a complete subgraph, by hypothesis, and so $a_{1} \ldots a_{i}$ and $a_{1} \ldots a_{i+2}$ are equal or adjacent in $X$. But then $a_{i+1} a_{i+2} \in \Sigma \cup\{1\}$, contradicting $\left(a_{1}, a_{2}, \ldots, a_{m}\right) \in L_{\Sigma}$.

Since $X_{\mathcal{C}}$ is a tree, there is only one geodesic from 1 to $g$. So there is only one word in $L_{\Sigma}$ representing $g$, and $(\Gamma, \Sigma)$ is a unique factorisation pair.

We conclude this section with a result mentioned in the introduction:

Proposition 2. If $(\Gamma, \Sigma)$ is a unique factorisation pair, then $X=\operatorname{Cay}(\Gamma, \Sigma)$ is 2-bounded by a tree.

Proof: Let $E_{0}$ denote the set of all edges $\left\{a_{1} \ldots a_{m-1}, a_{1} \ldots a_{m}\right\}$ of $X$, where $\left(a_{1}, \ldots, a_{m}\right) \in L_{\Sigma}$ has length $m \geqslant 1$. That is, $E_{0}$ consists of the edges $\{x, y\}$ of $X$ for which $d_{\Sigma}(1, x) \neq d_{\Sigma}(1, y)$. Now $X_{0}=\left(\Gamma, E_{0}\right)$ is a tree. For if $\pi=\left(g_{0}, \ldots, g_{n}=g_{0}\right)$ is a circuit in $X_{0}$, we may choose the numbering so that $d_{\Sigma}\left(1, g_{0}\right) \geqslant d_{\Sigma}\left(1, g_{i}\right)$ for $i=0, \ldots, n$. But $g_{0}$ has only one neighbour $g$ in $X_{0}$ satisfying $d_{\Sigma}(1, g)<d_{\Sigma}\left(1, g_{0}\right)$, namely $a_{1} \ldots a_{m-1}$ if $\left(a_{1}, \ldots, a_{m}\right)$ represents $g_{0}$. So $g_{1}=g_{n-1}$, in contradiction to $\pi$ being a circuit. So $X_{0}$ is a spanning tree for $X$.

Let $\{x, y\}$ be an edge in $X$. If $d_{\Sigma}(1, x) \neq d_{\Sigma}(1, y)$, then $\{x, y\} \in E_{0}$ and $d_{X_{0}}(x, y)=1$. If $d_{\Sigma}(1, x)=d_{\Sigma}(1, y)$, then $x=a_{1} \ldots a_{m}$ and $y=a_{1} \ldots a_{m} a$ for some $\left(a_{1}, \ldots, a_{m}\right) \in L_{\Sigma}$ and $a \in \Sigma$ such that $a_{m} a \in \Sigma$. So for $z=a_{1} \ldots a_{m-1},(x, z, y)$ is a path in $X_{0}$ of length 2, and so $d_{X_{0}}(x, y)=2$. So $X$ is 2 -bounded by a tree.

\section{REFERENCES}

[1] J. Avenhaus, K. Madlener and F. Otto, 'Groups presented by finite two-monadic Church-Rosser Thue systems', Trans. Amer. Math. Soc. 297 (1986), 427-443. 
[2] G. D'Agostino, 'Cayley graphs of virtually free groups', Internat. J. Algebra Comput. 3 (1993), 189-199.

[3] R.H. Haring-Smith, 'Groups and simple languages', Trans. Amer. Math. Soc. 279 (1983), 337-356.

[4] J. Mairesse, 'Random walks on groups and monoids with a Markovian harmonic measure', (preprint, 2004) Available at http://ww.liafa.jussieu.fr/"mairesse/Article/ and http://wru.liafa.jussieu.fr/web9/rapportrech/description_en.php? idrapportrech $=666$.

[5] J. Mairesse and F. Mathéus, 'Random walks on groups with a tree-like Cayley graph', in Mathematics and computer science. III. Algorithms, trees, combinatorics and probabilities pages 445-460, Trends Math. (Birkhäuser Verlag, Basel, 2004).

[6] M. Shapiro, 'Pascal's triangles in Abelian and hyperbolic groups', J. Austral. Math. Soc. Ser. A 63 (1997), 281-288.

[7] J. Stallings, 'A remark about the description of free products of groups', Proc. Cambridge Philos. Soc. 62 (1966), 129-134.

School of Mathematics and Statistics

The University of Sydney

New South Wales 2006

Australia

e-mail: donaldc@maths.usyd.edu.au

bernhard@maths.usyd.edu.au 